\title{
Estimación de gas metano mediante el modelo LandGEM del relleno sanitario municipal de residuos sólidos de Patate-Pelileo, Tungurahua, Ecuador Estimation of methane gas by LandGEM model from Patate-Pelileo municipal solid waste landfill, Tungurahua, Ecuador
}

Robinson Rigoberto Ambuludi-Paredes ${ }^{1}$, Victor Paul Carvajal-Padilla ${ }^{2}$, Karel Diéguez-Santana ${ }^{3}$

Fecha de recepción: 14 de octubre de 2020 Fecha de aprobación: 23 de enero de 2021

Ambuludi-Paredes, R.R; Carvajal-Padilla, V.P; DiéguezSantana, K. Estimación de gas metano mediante el modelo landgem del relleno sanitario municipal de residuos sólidos de patate-pelileo, Tungurahua, Ecuador. Tecnología en Marcha. Vol. 35-1. Enero-Marzo 2022. Pág 67-78.

doi) https://doi.org/10.18845/tm.v35i1.5413

1 Universidad Estatal Amazónica. Ecuador. Correo electrónico: ambuludi94@gmail.com

2 Universidad Estatal Amazónica. Ecuador. Correo electrónico: vpaulcp@gmail.com 


\title{
Palabras clave
}

Metano; contaminación atmosférica; eliminación de residuos; biogás; tratamiento de residuos; LandGEM.

\section{Resumen}

El crecimiento económico y demográfico de los países han incrementado la tasa de generación de residuos sólidos, y ha creado una nueva amenaza para la sostenibilidad. El objetivo principal de este estudio fue estimar el potencial de gas metano en el relleno sanitario de residuos sólidos de la mancomunidad Patate-Pelileo, Tungurahua, Ecuador mediante el modelo LandGEM, modificado a las condiciones de Ecuador. Para ello se utilizó información desde inicio del relleno, vida útil, clima, cantidad de residuos, composición de los residuos que fueron obtenidos de bases de datos y reportes estadísticos. Como resultado, se observa que la cantidad de metano calculada para los primeros 20 años fue de 33.070.236,19 $\mathrm{m}^{3}$, una media anual de 1.653.511,81 $\mathrm{m}^{3}$, y el valor máximo se alcanza en el año 2032 (1.985.270.108 $\mathrm{m}^{3}$ ). La electricidad total estimada de 2011 a 2032 oscila entre 17693 MWh y 53385 MWh, que puede suministrar los requerimientos del relleno sanitario y otras prestaciones. La investigación permite concluir que los modelos de estimación se pueden utilizar para la planificación de la producción de energía y otros usos del gas de relleno sanitario, pero tienen gran dependencia de la calidad de los datos disponibles, y de la selección de los coeficientes correctos. Además, están fuertemente influenciada por las cantidades de residuos generadas.

\section{Keywords}

Methane; air pollution; waste disposal; biogas; waste treatment; LandGEM.

\begin{abstract}
The economic and demographic growth of the countries has increased the rate of solid waste generation and created a new threat to sustainability. The main objective of this study was to estimate the methane gas potential in the solid waste landfill of the Patate-Pelileo community, Tungurahua, Ecuador using the LandGEM model modified to the conditions of Ecuador. Information from the beginning of the landfill, useful life, climate, amount of waste, waste composition was obtained from databases and statistical reports. As a result, it is observed that the total amount of methane calculated for the first 20 years was $33.070 .236,19 \mathrm{~m}^{3}$, an annual average of $1.653 .511,81 \mathrm{~m}^{3}$, and the maximum value is reached in the year $2032(1.985 .270,108$ $\left.\mathrm{m}^{3}\right)$. The total estimated electricity from 2011 to 2032 ranges from $17693 \mathrm{MWh}$ to $53385 \mathrm{MWh}$, which can supply the requirements of the landfill and other facilities. The research concludes that the estimation models can be used for planning energy production and other uses of landfill gas but are highly dependent on the quality of the available data, and the selection of the correct coefficients. Furthermore, they are strongly influenced by the amounts of waste generated.
\end{abstract}

\section{Introducción}

El crecimiento económico y demográfico de los países han incrementado la tasa de generación de residuos sólidos, y ha creado una nueva amenaza para la agenda global de sostenibilidad de la Organización de las Naciones Unidas (ONU) [1]. Nuevos lugares anteriormente con ecosistemas naturales han sido convertidos en hogares, infraestructuras o vertederos, para satisfacer las necesidades humanas. Este proceso se refleja en la generación de residuos en América Latina y el Caribe, pues en el 2014 se generaban diariamente 541.000 t/día (de ellos, 
145.000 t/día de residuos a basurales) y se estima para el año 2050, que las cifras lleguen a las 671.000 t/día. La tasa promedio de generación actual de la región es de 1,04kg/hab.-día, alrededor de 40 millones de personas carecen de acceso a la recolección de residuos y más del 50\% de los residuos sólidos urbanos (RSU) generados son orgánicos [2].

El aumento de las cantidades de residuos sólidos generados puede desencadenar graves problemas ambientales y afectar negativamente la vida humana desde un punto de vista ambiental, económico y de salud, por lo que la recolección y aprovechamiento de los residuos sólidos es una actividad muy importante para la sostenibilidad [3]. Sin embargo, la organización y planificación de los sistemas de gestión de RSU en países vías de desarrollo como Ecuador es muy deficiente. Existe falta de información sobre las cantidades y tipos de RSU recolectados, así como las cantidades que son reciclables y recuperables, adicionalmente no se han abordado profundamente el comportamiento de los sitios de disposición final, todos estos motivos conllevan a la búsqueda de alternativas de gestión eficientes, a tomar medidas de control sobre los residuos sólidos generados por las diferentes ciudades, y a reducir considerablemente la contaminación ambiental que estos producen.

Los sitios de disposición final de RSU son una fuente potencialmente importante de emisiones de gases de efecto invernadero (GEI), especialmente metano $\left(\mathrm{CH}_{4}\right)$, y dióxido de carbono $\left(\mathrm{CO}_{2}\right)$ a la atmósfera [4,5]. Si bien las emisiones de la mayoría de los gases de los rellenos sanitarios se han estabilizado en gran medida en los países desarrollados, las emisiones de los países en desarrollo han continuado en aumento[6].

En Ecuador, en el año 2018, según las estadísticas ambientales del Instituto Nacional de Estadísticas y Censos (INEC), un habitante de la zona urbana produce en promedio 0,86 kg de residuos sólidos al día, y se recolectaron 12.739 toneladas de residuos sólidos promedio diarias. Del total de residuos sólidos el $56,2 \%$ corresponde a residuos orgánicos y el 43,8\% a inorgánicos, de ellos $11,2 \%$ de plásticos y 9,1\% de cartón/papel, 6,7\% sanitario, y 15,6 de otras fracciones. Mientras, solo el 44,9\% de los Gobiernos Autónomos Descentralizados Municipales (GADM), disponen los residuos sólidos urbanos en rellenos sanitarios [7]. Según la Constitución del Ecuador (Artículo 264) los GADM tiene la competencia de prestar los servicios públicos de manejo de residuos sólidos y otras actividades de saneamiento ambiental. Por otra parte, como modelo de Gestión, el artículo 275 del Código Orgánico de Organización Territorial, Autonomía y Descentralización (COOTAD) permite que este servicio puede ser prestado de forma directa, por contrato, gestión compartida, por delegación a otro gobierno o por cogestión con la comunidad y empresas de economía mixta. En este sentido desde inicios del 2011 funciona la Empresa Municipal Mancomunada de los municipios de Patate y Pelileo (EMMAIT-EP) para el servicio del manejo integral de los residuos sólidos en estos municipios de la provincia Tungurahua, Ecuador.

Por otra parte, el Ministerio del Ambiente, mediante el Programa Nacional para la Gestión Integral de Desechos Sólidos (PNGIDS), en funcionamiento desde el año 2010 ha impulsado estrategias para mejorar los planes de operación y gestión de los RSU, pero si bien se ha intentado potenciar estrategias para prevenir, minimizar la generación, aumentar las tasas de reciclaje, tratamiento o aprovechamiento, aún los RSU son mayoritariamente depositados en los sitios definidos por cada municipio para ello [8]. Por lo que proponer soluciones de gestión sostenible con respecto a su disposición final permitirá obtener criterios que reducirán los impactos ambientales asociados con la gestión de RSU [9].

En Ecuador, apenas existe información en la literatura que identifique y cuantifique las emisiones de GEl de los sitios de disposición final. Aun siendo mayormente condiciones tropicales (temperaturas y la humedades generalmente altas), que propician la degradación anaeróbica de los residuos y por ende, resulta en cantidades potencialmente grandes de $\mathrm{CH}_{4}$ 
como la principal emisión [10]. La cuantificación de las emisiones de gases del relleno sanitario, son el 'primer paso para búsqueda de alternativas que conlleve a la reducción de las emisiones atmosféricas y a la generación de energía limpia y renovable [11].

Para aportar más información sobre la gestión de residuos en el contexto ecuatoriano, el objetivo de este estudio fue estimar el potencial de gas metano mediante el modelo LandGEM, modificado a las condiciones de Ecuador, en el relleno sanitario de residuos sólidos de la mancomunidad Patate-Pelileo, Tungurahua, Ecuador. El estudio también recolectará información primaria y secundaria de los residuos sólidos depositados en el relleno sanitario de la mancomunidad Patate-Pelileo y describirá criterios de la planificación e implementación de actividades relevantes de gestión de residuos en el área de estudio.

\section{Materiales y métodos}

\section{Área de estudio}

La investigación se desarrolló en la ciudad de Pelileo, cantón de la provincia de Tungurahua, en el relleno sanitario de la mancomunidad Patate-Pelileo, que cuenta con una extensión de 3,5 ha de las cuales 2 ha se encuentran ocupadas, se ubica en el km 3 de la vía Patate-Pelileo, en las coordenadas $775884.47 \mathrm{mE}, 9852807.64 \mathrm{mS}$ a 2344 m.s.n.m. El sitio posee una humedad relativa del $65 \%$ y una temperatura promedio de $18^{\circ} \mathrm{C}$, su precipitación promedio anual oscila los $700 \mathrm{~mm}$, siendo los meses de abril y mayo los de mayor presencia de lluvias, mientras, la temporada más seca se presenta en los meses de junio a octubre. Los suelos son de origen Inceptisoles, de color negro, arenosos [12]. La figura 1 muestra la ubicación geográfica del relleno sanitario y área de estudio.

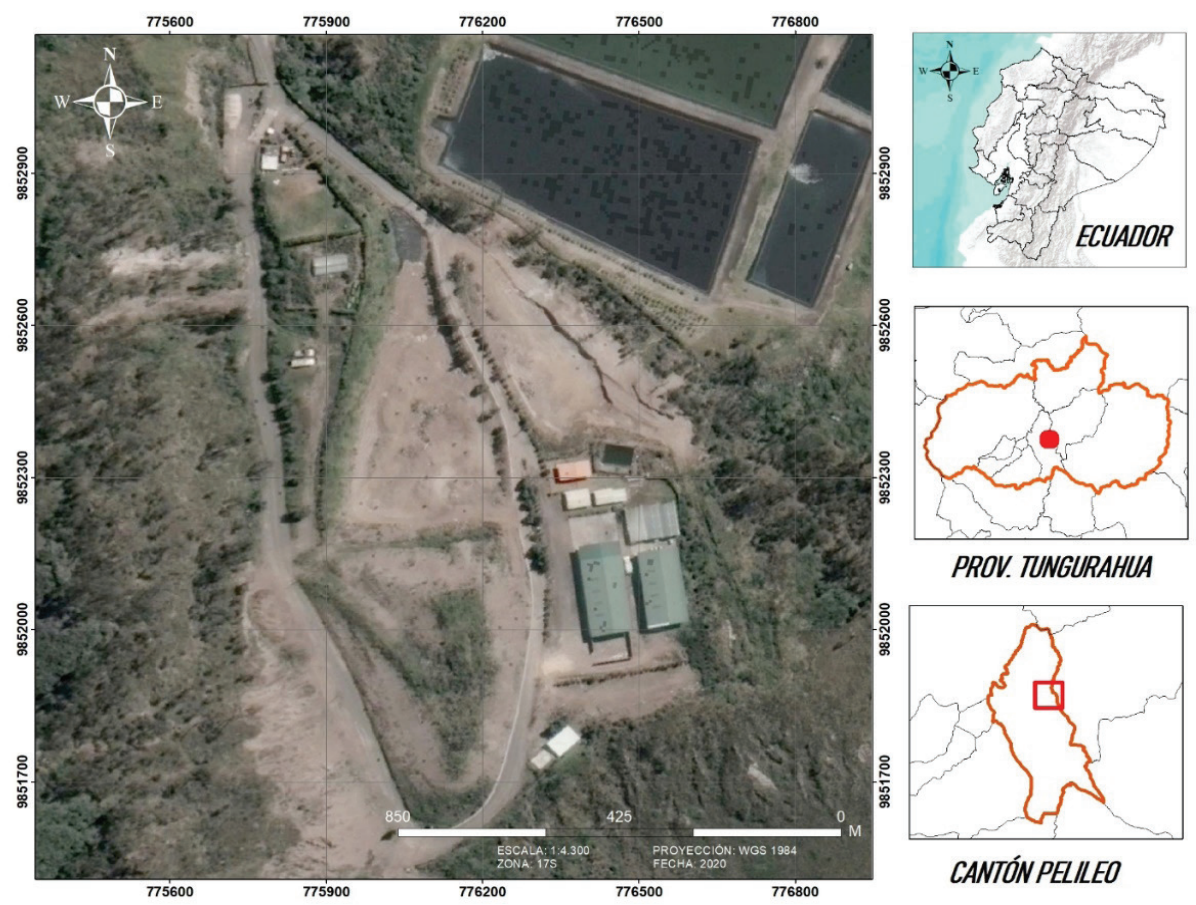

Figura 1. Ubicación del relleno sanitario de la mancomunidad Patate-Pelileo (EMMAIT-EP). 


\section{Recopilación de datos}

Para estimar la producción de metano a partir de los RSU se empleó el modelo matemático LandGEM, los parámetros del modelo se seleccionaron acorde a las condiciones del relleno sanitario de la mancomunidad de Patate-Pelileo. Se tomaron variables como: clima, humedad, precipitación, cantidad de residuos, producción per cápita, porcentaje de residuos orgánicos e inorgánicos, etc. Estos fueron obtenidos de bases de datos, reportes y estadísticas específicas. En el caso del clima del Instituto Nacional de Meteorología e Hidrología (INAMHI). Mientras los datos de los RSU (información de la composición y las cantidad de los residuos sólidos generados), se obtuvieron de datos proporcionados directamente por EMMAIT-EP, empresa encargada de la administración del relleno sanitario de la mancomunidad Patate Pelileo. Estos fueron complementados por las estadísticas nacionales como: INEC y la Asociación de Municipalidades del Ecuador (AME).

Para el cálculo de la producción per cápita y la proyección de la población, también se emplearon datos relacionados con el relleno sanitario de la mancomunidad Patate-Pelileo y los datos demográficos se recopilaron en función de la tasa de crecimiento anual de la población hasta el 2031, pues el relleno sanitario comenzó a operar en el año 2011, y fue diseñado para un tiempo de vida útil del relleno sanitario de 20 años [29], por lo que para el estudio se debe considerar la generación de residuos hasta ese año.

Para calcular la producción per cápita (ppc) de residuos se utilizó la ecuación 1.

$$
\mathrm{PPC}=\frac{\mathrm{W}}{\mathrm{P}}
$$

Donde:

- PPC: Producción per cápita de residuos sólidos, kg/hab*día.

- W: Peso generado de residuos sólidos en un día, kg.

- P: Población que generó esos residuos sólidos en un día, habitantes

Mientras, para la estimación de la población futura, se consideró la ecuación 2, y se tomó de las fuentes estadísticas de INEC, la tasa de crecimiento poblacional

$$
P_{f}=P_{i} *(1+r)^{t}
$$

Donde:

- $\quad P_{f}$ : Población futura

- $P_{i}$ : Población inicial

- $r$ : tasa de crecimiento

- $t$ : tiempo en años a los que se va a proyectar

\section{Modelo LandGEM}

Para calcular la cantidad de emisiones de metano se utilizó el modelo de LandGEM, herramienta basada en una ecuación de primer orden de la tasa de descomposición de residuos en una cantidad estimada de metano que se generará en un período específico de tiempo (año) $[13,14]$. Este modelo de la EPA (United States Environmental Protection Agency, Agencia de Protección Ambiental de Estados Unidos), está basada en una hoja de cálculo Excel que fue adaptada a las condiciones de Ecuador. La generación de biogás, se estima en función del contenido de metano del biogás, en este caso se consideró un 50\% [15]. Las entradas para el 
cálculo en el modelo LandGEM incluyen (1) área y capacidad del relleno sanitario, (2) cantidad anual de residuos recibidos, (3) constante de tasa de generación de metano ( $k$ ) y potencial de generación de metano (Lo), y (4) años totales de relleno sanitario operación [16]

$$
Q_{C H_{4}}=\sum_{i=1}^{n} \sum_{j=0.1}^{1} k * L_{0}\left(\frac{M_{i}}{10}\right) * e^{-k T_{i j}}
$$

Donde:

- $Q \mathrm{CH}_{4}$ : es la generación anual de $\mathrm{CH}_{4}$ en el año del cálculo ( $\mathrm{m}^{3}$ / año),

- $i$ : es el incremento de tiempo de 1 año,

- j: es el incremento de tiempo de 0.1 años,

- $n$ : es el (año del cálculo) (año inicial de aceptación de residuos),

- $k$ : es la constante generación de $\mathrm{CH}_{4}$ (año 1),

- $L_{0}$ es la capacidad potencial de generación de $\mathrm{CH}_{4}\left(\mathrm{~m}^{3} / \mathrm{t}\right)$,

- Mi: es la cantidad de residuos recibidos en el año i (t),

- $T_{1 J}$ : es la edad de la sección j de cantidad de residuos Mi aceptado en el año i.

El modelo LandGem se adaptó a las condiciones climáticas de Ecuador y las variables Índice de generación de metano $(k)$, Índice de generación potencial de metano $\left(L_{0}\right)$ enfocadas a las condiciones del relleno sanitario, nivel de precipitaciones y a la cantidad de residuos orgánicos presentes en el lugar de estudio. El cuadro 1 muestra los valores de $\mathrm{k}$ y $\mathrm{L}_{0}$.

Cuadro 1. Descripción de los valores de $k$ y $L_{0}$ tomando en cuenta la precipitación del lugar de estudio y la composición de residuos.

\begin{tabular}{|c|c|c|c|c|}
\hline Precipitación (mm/año) & $\begin{array}{c}k \text { (Residuos } \\
\text { orgánicos } \leq 50)\end{array}$ & $\begin{array}{c}k \text { (Residuos } \\
\text { orgánicos } \geq 60\end{array}$ & $\begin{array}{c}\text { Lo (Residuos } \\
\text { orgánicos } \leq 50)\end{array}$ & $\begin{array}{c}\text { Lo (Residuos } \\
\text { orgánicos } \geq 60\end{array}$ \\
\hline $0-249$ & 0,4 & 0,43 & 60 & 62 \\
\hline $250-499$ & 0,5 & 0,53 & 80 & 83 \\
\hline $500-999$ & 0,65 & 0,69 & 84 & 87 \\
\hline $1000-1999$ & 0,8 & 0,85 & 84 & 87 \\
\hline+2000 & 0,8 & 0,85 & 84 & 87 \\
\hline
\end{tabular}

Fuente: [17]

Para el presente estudio se consideró un valor de $k=0,69$ y de $L_{0}=87$, respectivamente, debido al clima frío con precipitación promedio del lugar de estudio de 700 mm/año [18], y la cantidad de residuos orgánicos de alimentos son superiores al 60\% [7].

\section{Determinación del potencial de generación de electricidad}

El metano producido a partir de fracciones de residuos se emplea para determinar el potencial de generación de electricidad. La ecuación 4 muestra como calcular el potencial de generación de electricidad de la sistemas de captación de biogás de rellenos sanitarios. 


$$
E A G=\frac{Q_{C H 4} * 37.2 * E G e * E R * 0.9 * C}{\emptyset}
$$

Dónde:

EAG: es la electricidad anual generada (MWh),

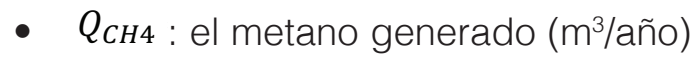

- $37.2\left(\mathrm{MJ} / \mathrm{m}^{3}\right)$ es el valor de calentamiento más bajo de metano [19, 20]

- EGe: es la eficiencia de generación de electricidad del dispositivo de conversión, según varios autores se puede considerar de 35\% [20, 21].

- ER: es la eficiencia de recolección de metano, se consideró de 75\% como plantean [20, 22].

- 0.9 es el factor de oxidación del relleno sanitario [19].

- C es el factor de capacidad, y se considera como 85\% [20, 23], y

- $\varnothing$ es el factor de conversión de MJ a MWh.

\section{Resultados}

Recopilación de información primaria y secundaria de los residuos sólidos depositados en el relleno sanitario de la mancomunidad Patate-Pelileo.

Según los datos disponibles en las oficinas de EMMAIT-EP y la proyección de la cantidad de residuos sólidos a ingresar al relleno sanitario del estudio de Castro [24] y los datos recopilados por el INEC [7], los residuos sólidos depositados en el relleno sanitario de la mancomunidad Patate-Pelileo, tienen las características que expone el cuadro 2.

Cuadro 2. Composición de los RSU que son depositados en el relleno sanitario de la mancomunidad Patate-Pelileo.

\begin{tabular}{|c|c|c|c|}
\hline Componentes de los residuos & Promedio (\%) & Componentes de los residuos & Promedio (\%) \\
\hline Residuos orgánicos & 66,01 & Metales ferrosos & 2,14 \\
\hline Plásticos & 9,16 & Residuos de jardinería & 1,41 \\
\hline Pañales & 8,24 & Caucho & 1 \\
\hline Textiles & 3,85 & Vidrio & 0,97 \\
\hline Cartón & 3,72 & Metales no ferrosos & 0,34 \\
\hline Papel & 2,26 & Otros & 0,9 \\
\hline
\end{tabular}

Fuente: [7]

Como se muestra en el cuadro 2, los resultados obtenidos de la composición de los RSU que ingresan al relleno sanitario Patate-Pelileo, refleja que la fracción mayoritaria es residuos de alimentos, seguido de plásticos, pañales, textiles, papel y cartón.

Por su parte, el cuadro 3 registra el comportamiento de la generación de RSU y de la población hasta la fecha, y la proyección de los próximos años hasta el 2031 (fecha de vida útil del relleno sanitario).

Tomando en consideración que la fecha de inicio de operación del relleno sanitario de la mancomunidad Patate-Pelileo fue en el año 2011, el tiempo de vida útil del relleno sanitario de 20 años [24], la ppc (producción per cápita) actual y sus datos históricos, se obtuvo una 
proyección hasta el año 2031, dando un total de 389.489,31 toneladas de residuos sólidos municipales, y una ppc de 0,76 kg/hab*día. A partir del año 2020 se proyectó la generación anual considerando un incremento de la generación y de la población, este último según la tasa de crecimiento anual.

Cuadro 3. Proyección de residuos sólidos del relleno sanitario de la mancomunidad Patate-Pelileo.

\begin{tabular}{|c|c|c|c|}
\hline Año & Generación de RSU, t/día & $\begin{array}{c}\text { Generación de RSU } \\
(\mathrm{t} / \mathrm{a})\end{array}$ & ppc (kg/hab*día) \\
\hline 2011 & 40,17 & $14.662,05$ & 0,61 \\
\hline 2012 & 41,20 & $15.038,00$ & 0,62 \\
\hline 2013 & 42,25 & $15.421,25$ & 0,63 \\
\hline 2014 & 43,33 & $15.815,45$ & 0,63 \\
\hline 2015 & 44,44 & $16.220,60$ & 0,64 \\
\hline 2016 & 45,48 & $16.599,83$ & 0,65 \\
\hline 2017 & 46,55 & $16.989,29$ & 0,65 \\
\hline 2018 & 47,61 & $17.378,74$ & 0,66 \\
\hline 2019 & 48,68 & $17.768,20$ & 0,67 \\
\hline 2020 & $49,75 p$ & $18.157,65 p$ & $0,67 p$ \\
\hline 2021 & $50,81 p$ & $18.547,11 p$ & $0,68 p$ \\
\hline 2022 & $51,88 p$ & $18.936,56 p$ & $0,69 p$ \\
\hline 2023 & $52,95 p$ & $19.326,02 p$ & $0,69 p$ \\
\hline 2024 & $54,01 p$ & $19.715,47 p$ & $0,71 p$ \\
\hline 2025 & $55,08 p$ & $20.104,93 p$ & $0,71 p$ \\
\hline 2026 & $56,15 p$ & $20.494,38 p$ & $0,72 p$ \\
\hline 2027 & $57,22 p$ & $20.883,84 p$ & $0,73 p$ \\
\hline 2028 & $58,28 p$ & $21.273,29 p$ & $0,73 p$ \\
\hline 2029 & $59,35 p$ & $21.662,75 p$ & $0,74 p$ \\
\hline 2030 & $60,42 p$ & $22.052,20 p$ & $0,75 p$ \\
\hline 2031 & $61,48 p$ & $22.441,66 p$ & $0,76 p$ \\
\hline TOTAL & & $389.489,31$ & *p (proyección) \\
\hline
\end{tabular}

\section{Cálculo de producción de metano con el modelo Landgem}

Una vez recopilada y calculada la información inicial, se procedió a estimar la producción del metano del relleno sanitario de la mancomunidad Patate-Pelileo, mediante la metodología (LandGem modificado para Ecuador) el volumen total de metano de 33.070.236,19 $\mathrm{m}^{3}$, para una media anual de1.653.511,81 $\mathrm{m}^{3}$. La figura 2 muestra la producción anual, como se puede observar el valor más alto de producción de gas del relleno sanitario es un año después del cierre (Año 2032, 1.985.270,11 m³) esto es debido a que es año posterior al mayor ingreso de residuos proyectado, toda la materia orgánica se degradaría para esa fecha por las condiciones del relleno sanitario. El crecimiento exponencial en los primeros años de funcionamiento, es 
debido a la cantidad de materia orgánica que ingresa en el relleno sanitario comportamiento similar al estudio realizado por Biglari, et al. [25], donde el pico más alto es dos años después del cierre y la producción de gas del relleno sanitario se reduce exponencialmente para los años siguientes.

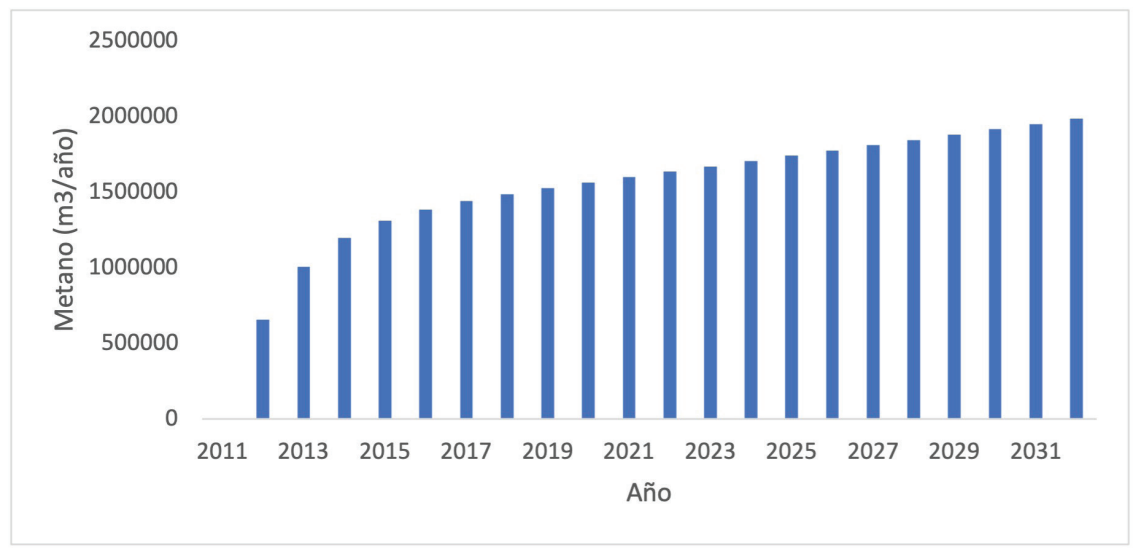

Figura 2. Producción de metano anual del relleno sanitario de la mancomunidad Patate-Pelileo, período 2011-2032 ( $\mathrm{m}^{3} / \mathrm{año}$ ).

Mientras que la figura 3 muestra la tendencia de las emisiones gaseosas anuales (Mg/año) de todos los gases estimados (Gas total de relleno sanitario, metano y dióxido de carbono). Como se puede apreciar la producción anual aumenta hasta un año después del cierre del relleno sanitario, a partir de ese año comienzan a descender, esto está asociado a que mientras se estén ingresando residuos se incrementa la generación, como la fracción mayoritaria de los residuos eliminados en el relleno sanitario son restos de alimentos, su vida en la producción de metano es corta, por lo que una vez cerrado el relleno, comienza el descenso de la generación de gases [26].

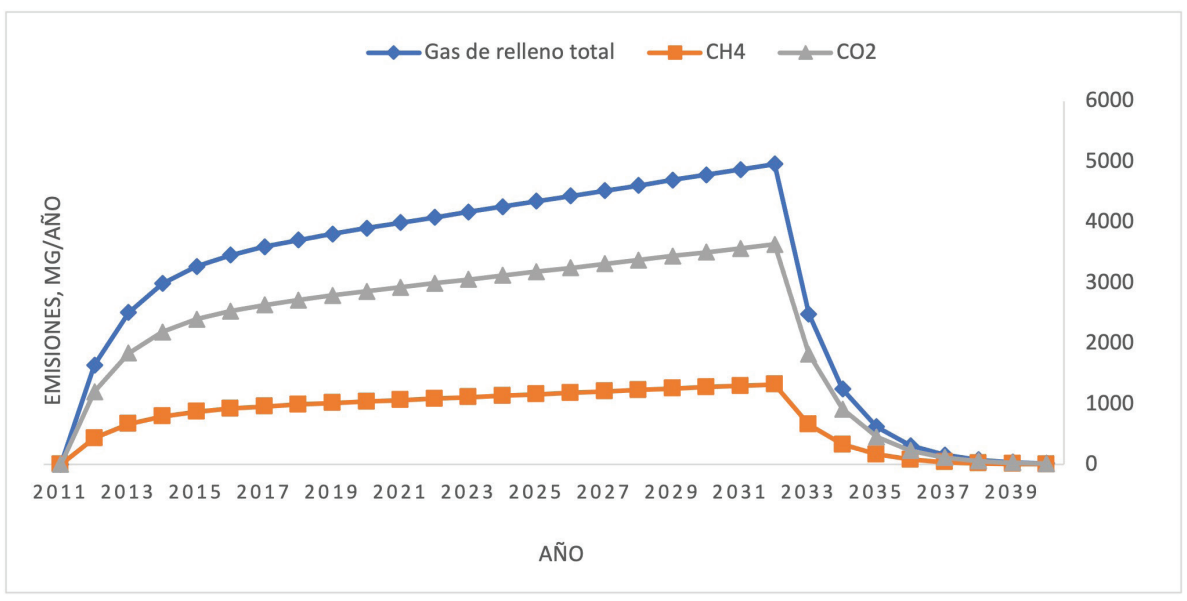

Figura 3. Emisiones gaseosas anuales basados en el software LANDGEM para el relleno sanitario Patate-Pelileo, periodo 2011-2040, (Mg/Año)

En el cuadro 4 se puede observar la comparación de este estudio con otros resultados obtenidos en distintos países del área. Como similitud todos los estudios emplearon metodologías basadas en el modelo LandGem. En el caso del estudio realizado por Escamilla-García, et al. [14] en la ciudad de Oaxaca, México, utilizan el modelo de LandGem modificado para México, y obtienen

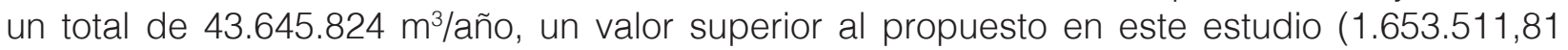


$\mathrm{m}^{3}$ (año), pero ese estudio fue realizado para una ciudad mayor y el relleno sanitario recibe cantidades más elevadas de RSU que al de la Mancomunidad Patate-Pelileo. Mientras, el estudio realizado por Pérez, et al. [27] en la ciudad de Juliaca San Román, Puno, Perú, solo emplean el el modelo LandGEM, versión 3.02 con los parámetros pre establecidos. De igual manera, obtienen un valor de 29.925.493,1 $\mathrm{m}^{3} /$ año, que es mucho mayor al de este trabajo, aunque reciben 198,26 t/día, mientras que en Patate-Pelileo, Ecuador solo ingresan 48,68 t/ día. Aun así los resultados son correspondientes, pues como menciona [17], la producción de metano es menor debido a la menor población y cantidad de residuos producidos.

Cuadro 4. Resultados y comparación con otros estudios de producción estimada de metano con el modelo de LandGem.

\begin{tabular}{|c|c|c|c|c|c|c|}
\hline $\begin{array}{l}\text { Lugar (Ciudad, } \\
\text { país) }\end{array}$ & $\begin{array}{l}\text { Población, } \\
\text { habitantes }\end{array}$ & $\begin{array}{l}\text { ppc (kg/ } \\
\text { hab*día) }\end{array}$ & $\begin{array}{l}\text { Generación de } \\
\text { residuos, t/día }\end{array}$ & $\begin{array}{l}\text { Fracción de } \\
\text { orgánicos, \% }\end{array}$ & $\begin{array}{l}\text { Producción } \\
\text { de } \mathrm{CH}_{4}\left(\mathrm{~m}^{3} /\right. \\
\text { año) }\end{array}$ & Referencia \\
\hline $\begin{array}{c}\text { Juliaca, San } \\
\text { Román, Puno, Perú }\end{array}$ & 225.146 & 0,72 & 198,26 & 54,19 & $29.925 .493,1$ & [27] \\
\hline Oaxaca, México & 3.967 .889 & 0,41 & $1.661,5$ & 51,3 & 43.645 .824 & [14] \\
\hline $\begin{array}{l}\text { Patate-Pelileo, } \\
\text { Ecuador }\end{array}$ & 722.657 & 0,67 & 48,68 & 66,01 & $1.653 .511,81$ & Este estudio \\
\hline
\end{tabular}

Determinación del potencial de generación de electricidad

Se evaluó el potencial de generación de electricidad del biogás a partir de la fracción orgánica de los desechos sólidos urbanos recolectados para su eliminación en el relleno sanitario de la mancomunidad Patate-Pelileo. La figura 4 muestra los resultados de los potenciales de generación de electricidad. Como se puede apreciar existe un adecuado potencial de generación de electricidad en el relleno sanitario de la mancomunidad. La electricidad total estimada de 2011 a 2032 oscila entre 17693 MWh y 53385 MWh, respectivamente. Estos valores son inferiores a otros estudios realizados previamente en otras regiones, por ejemplo, en México, [20, 21] en Nigeria y China, respectivamente, donde se generó una cantidad de electricidad superior, pero como plantean $[20,21]$ la generación de electricidad está asociada a la generación de desechos sólidos municipales que a la vez depende del aumento de la población y el desarrollo económico de cada región.

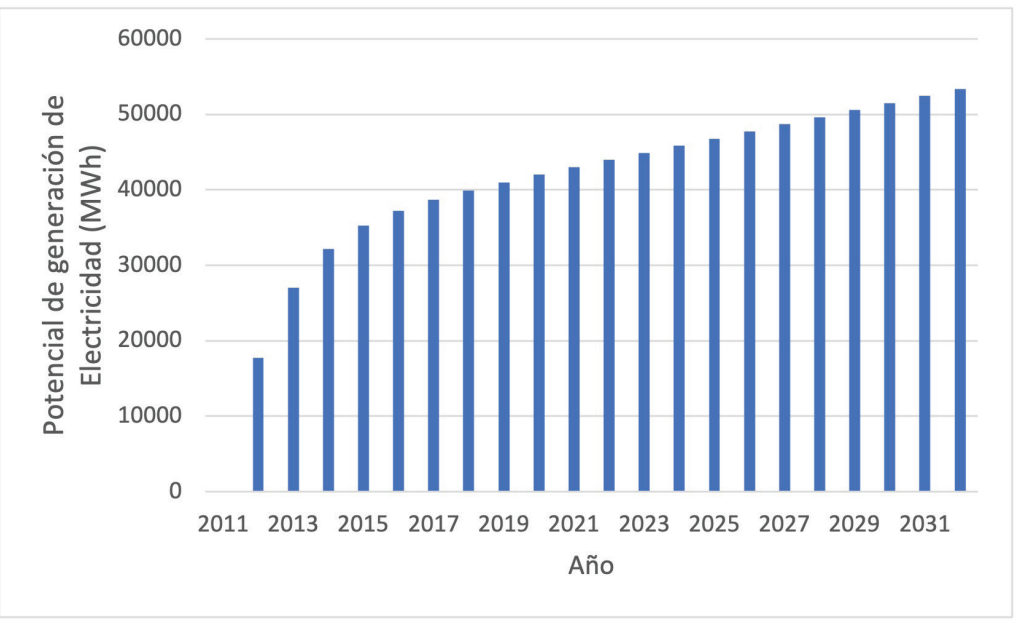

Figura 4. Potencial de generacion de electricidad (MWh) de las fracciones de residuos depositadas en el relleno sanitario mancomunidad Patate-Pelileo. 


\section{Conclusiones y recomendaciones}

La información primaria recolectada de los residuos sólidos depositados en el relleno sanitario de la mancomunidad Patate-Pelileo permitió estimar la generación de gases del relleno sanitario utilizando el modelo LandGEM para el relleno sanitario de la mancomunidad Patate-Pelileo, Tungurahua, Ecuador. Este relleno sanitario opera desde 2011 y debe cerrar en el 2031. La cantidad de metano calculada a partir de residuos sólidos fue de 33.070.236,19 $\mathrm{m}^{3}$, con una media de 1.653.511,81 m³/año para los primeros 20 años, el valor máximo se alcanza en el año 2032, de 1.985.270,108 m³/año. La electricidad total estimada de 2011 a 2032 oscila entre 17693 MWh y 53385 MWh, respectivamente que puede suministrar los requerimientos del relleno sanitario (sistemas de bombeo y tratamiento de lixiviados) y cantidades adicionales ser distribuidas a núcleos de hogares y otras prestaciones. Esta estimación tiene gran dependencia de la calidad de los datos disponibles y la selección de los coeficientes correctos. La producción estimada por este modelo está fuertemente influenciada por las cantidades de residuos generadas, por lo que aumentos de las futuras tasas de producción de residuos, también aumentara la producción de metano.

Los modelos para la estimación de metano de los rellenos sanitarios son fundamentales para considerar el posible impacto que puedan generar al aire, así como también ayudan a mantener un inventario de los gases de efecto invernadero y a llevar un control sobre los mismos, sin embargo para obtener resultados realistas, la elección de los parámetros de cada modelo debe ser escogido en dependencia del estado y las características del relleno sanitario, teniendo en consideración que aquel modelo que tome más parámetros relacionados con las cualidades intrínsecas del relleno sanitario será el que más se acerque a la estimación real de generación de metano.

\section{Referencias}

[1] O. O. Ayeleru, F. N. Okonta, and F. Ntuli, "Municipal solid waste generation and characterization in the City of Johannesburg: A pathway for the implementation of zero waste," Waste Management, vol. 79, pp. 87-97, 2018. https://doi.org/10.1016/j.wasman.2018.07.026.

[2] U. Environment, Waste Management Outlook for Latin America and the Caribbean. Panama City, Panama: United Nations Environment Programme. Latin America and the Caribbean Office, 2018.

[3] K. Diéguez-Santana, L. B. Sarduy-Pereira, and M. Decker, "Characterization and quantification of municipal solid waste in rural areas of the Ecuadorian Amazon," J. Environ. Treat. Tech., vol. 8, no. 4, 2020.

[4] N. Yang, H. Zhang, L.-M. Shao, F. Lü, and P.-J. He, "Greenhouse gas emissions during MSW landfilling in China: Influence of waste characteristics and LFG treatment measures," Journal of Environmental Management, vol. 129, pp. 510-521, 2013/11/15/ 2013. https://doi.org/10.1016/j.jenvman.2013.08.039.

[5] K. Diéguez-Santana, Y. Casas-Ledón, J. A. Loureiro-Salabarria, A. Pérez-Martínez, and L. E. Arteaga-Pérez, "A life cycle assessment of bread production: A Cuban case study," Journal of Environmental Accounting and Management, vol. 8, no. 2, pp. 125-137, 2020. https://doi.org/10.5890/JEAM.2020.06.002.

[6] A. I. Soto-Cabrera, A. P. Panimboza-Ojeda, C. G. Ilibay-Granda, C. R. Valverde-Lara, and K. Diéguez-Santana, "Impacto ambiental de la operación del Centro de faenamiento de la ciudad de Puyo, Pastaza, Ecuador," Prospectiva, vol. 18, no. 1, pp. 60-68, 2020. https://doi.org/10.15665/rp.v18i1.2101.

[7] INEC-AME. (2020, julio 30). Estadísticas de Información Ambiental Económica en Gobiernos Autónomos Descentralizados Municipales. Gestión de Residuos Sólidos 2018. Available: https://www.ecuadorencifras.gob.ec/documentos/web-inec/Encuestas Ambientales/Municipios 2018/Residuos solidos 2018/ PRESENTACION\%20RESIDUOS_2018.pdf

[8] MAE, "Informe de gestión MAE-PNGIDS 2010-2013. Programa Nacional de Gestión Integral de Desechos Sólidos.," 2014.

[9] T. P. Ramos-Ramos, D. J. Guevara-Llerena, L. B. Sarduy-Pereira, and K. Diéguez-Santana, "Producción más limpia y ecoeficiencia en el procesado del cacao. Un caso de estudio en Ecuador," Revista Investigación \& Desarrollo, vol. 20, no. 1, pp. 135-146, 2020. 10.23881/idupbo.020.1-10i. 
[10] N. M. Ngwabie, Y. L. Wirlen, G. S. Yinda, and A. C. VanderZaag, "Quantifying greenhouse gas emissions from municipal solid waste dumpsites in Cameroon," Waste Management, vol. 87, pp. 947-953, 2019/03/15/ 2019. https://doi.org/10.1016/i.wasman.2018.02.048.

[11] A. I. Soto-Cabrera, A. P. Panimboza-Ojeda, A. Ramones-Pinargote, A. Pérez-Martínez, L. B. Sarduy-Pereira, and K. Diéguez-Santana, "Huella de carbono en el cultivo de la caña de azúcar. Evaluación agrícola de un caso de estudio de la amazonia ecuatoriana," Ingenio Magno, vol. 11, no. 1, pp. 22-32, 2020.

[12] G. A. D. Tungurahua, "Plan de Ordenamiento territorial del cantón Pelileo," Pelileo, Tungurahua2019, Available: http://app.sni.gob.ec/sni-link/sni/PORTAL SNI/data sigad plus/sigadplusdocumentofinal/1860000640001 ACTUALPDYOT2015 15-03-2015 21-58-23.pdf, Accessed on: 2020/julio/30.

[13] D. Andriani and T. D. Atmaja, "The potentials of landfill gas production: a review on municipal solid waste management in Indonesia," Journal of Material Cycles and Waste Management, vol. 21, no. 6, pp. 1572-1586, 2019. https://doi.org/10.1007/s10163-019-00895-5.

[14] P. E. Escamilla-García, M. E. Jiménez-Castañeda, E. Fernández-Rodríguez, and S. Galicia-Villanueva, "Feasibility of energy generation by methane emissions from a landfill in southern Mexico," Journal of Material Cycles and Waste Management, vol. 22, no. 1, pp. 295-303, 2020. https://doi.org/10.1007/s10163-019-009403.

[15] LandGEM, "Landfill Gas Emissions Model," version 3.02. ed, 2005.

[16] H. Kamalan, M. Sabour, and N. Shariatmad, "A review on available landfill gas models," J Environ Sci Technol, vol. 4, pp. 79-92, 2011. https://doi.org/10.3923/jest.2011.79.92.

[17] S. Fallahizadeh, M. Rahmatinia, Z. Mohammadi, M. Vaezzadeh, A. Tajamiri, and H. J. M. Soleimani, "Estimation of methane gas by LandGEM model from Yasuj municipal solid waste landfill, Iran," vol. 6, pp. 391-398, 2019. https://doi.org/10.1016/i.mex.2019.02.013.

[18] INAMHI, "Anuario Meteorológico," vol. 202012, 2015.

[19] T. R. Ayodele, A. S. O. Ogunjuyigbe, and M. A. Alao, "Life cycle assessment of waste-to-energy (WtE) technologies for electricity generation using municipal solid waste in Nigeria," Applied Energy, vol. 201, pp. 200-218, 2017/09/01/ 2017. https://doi.org/10.1016/.j.apenergy.2017.05.097.

[20] D. Cudjoe, M. S. Han, and A. P. Nandiwardhana, "Electricity generation using biogas from organic fraction of municipal solid waste generated in provinces of China: Techno-economic and environmental impact analysis," Fuel Processing Technology, vol. 203, p. 106381, 2020/06/15/ 2020. https://doi.org/10.1016/j. fuproc.2020.106381.

[21] T. R. Ayodele, A. S. O. Ogunjuyigbe, and M. A. Alao, "Economic and environmental assessment of electricity generation using biogas from organic fraction of municipal solid waste for the city of Ibadan, Nigeria," Journal of Cleaner Production, vol. 203, pp. 718-735, 2018/12/01/ 2018. https://doi.org/10.1016/j.jclepro.2018.08.282.

[22] H. R. Amini, D. R. Reinhart, and A. Niskanen, "Comparison of first-order-decay modeled and actual field measured municipal solid waste landfill methane data," Waste Management, vol. 33, no. 12, pp. 2720-2728, 2013/12/01/ 2013. https://doi.org/10.1016/i.wasman.2013.07.025.

[23] IRENA, "Renewable Energy Technologies: Cost Analysis Series of Biomass for Power Generation,," Bonn. Germany2012, Available: https://www.irena.org/publications/2012/Jun/Renewable-Energy-Cost-Analysis--Biomass-for-Power-Generation.

[24] P. A. Castro, "Estudio de Impacto Ambiental ex- post para el "Relleno Sanitario EMMAIT-EP", provincia de Tungurahua, Cantones Pelileo y Patate. Ecuador "Ingeniería Geólogica, Minera y Ambiental Seguridad Y Salud Ocupacional, 2019.

[25] H. Biglari et al., "Estimating the amount of methane gas generated from the solid waste using the land GEM software, sistan and baluchistan," Journal of Global Pharma Technology., vol. 9, no. 3, pp. 35-41, 2017.

[26] T. Tsatsarelis and A. Karagiannidis, "Estimation of future methane production from Hellenic landfills," Global NEST Journal, vol. 11, no. 2, pp. 162-171, 2009. https://doi.org/10.30955/gnj.000591.

[27] G. B. M. Pérez, J. J. Q. Saavedra, and Y. M. V. Huaman, "Calculo de la generación de biogás para el relleno sanitario de la ciudad de Juliaca, utilizando el modelo LandGEM Versión 3.02 de la USEPA y estimación del potencial de producción eléctrica," Revista de Investigación Ciencia, Tecnología y Desarrollo, vol. 4, no. 2, pp. 42-55, 2018. https://doi.org/10.17162/rictd.v4i2.1096. 